\section{Beneficial Response to Phosphate Lowering Therapy in Normophosphatemic Tumoral Calcinosis}

Calcinosis cutis is a comprehensive terminology for disorders characterised by deposition of calcium salts in the cutaneous and subcutaneous tissue. Based on etiology, it can be classified into: dystrophic, metastatic, idiopathic, iatrogenic, and calciphylaxis. Dystrophic calcinosis occurs following a necrotic process unrelated to serum calcium level. Metastatic calcification results from precipitation of calcium salts due to high calcium or phosphorus levels. Calciphylaxis denotes calcification of the small and medium-sized blood vessels, of the dermis or subcutaneous tissue usually in the setting of renal failure [1]. Tumoral calcinosis refers to a severe form of calcinosis involving deeper tissues, especially around joints leading to limitation of movement.

A 14-year-old boy presented with history of hard swellings in the skin around multiple joints for past two years. Some of them ulcerated to extrude chalky white material. There was restriction of movements at knees, shoulders and elbows. He did not have history or examination findings to suggest autoimmune connective tissue disorders, pancreatic disease, chronic renal failure, malignancy, trauma, medical intervention in the affected regions. His previous records showed normal calcium and phosphorus levels, and he was not on calcium or vitamin D supplementation. There was no family history of similar condition. He had undergone excision of a large lesion from right axilla but did not receive any oral or parenteral medications for treatment of lesions before presenting to us.

There were multiple hard swellings involving the skin and subcutaneous tissue around shoulder, elbow, hip, knee joint and neck on both sides (Fig. 1a). Range of movement of all large joints measured using a universal goniometer showed restriction of joint movements which was maximum at right knee joint (50 degrees) and right elbow (30 degrees) for extension. Restriction of movements at the knee joints had resulted in limping. The physical examination was otherwise unremarkable.

Blood counts, renal function, liver function parameters were normal. Average serum calcium and phosphorus level(fasting sample) after multiple measurements were normal $(9.4 \mathrm{mg} / \mathrm{dL}$ and $4.8 \mathrm{mg} / \mathrm{dL}$, respectively).Vitamin D insufficiency (25-hydroxy vitamin D level $12 \mathrm{ng} / \mathrm{mL}$ ) and secondary hyperparathyroidism (Serum PTH level $126 \mathrm{pg} / \mathrm{mL}$ ) were present. Plasma levels of 1, 25- dihydroxy Vitamin D was normal. Antinuclear antibody (ANA), Anti ds-DNA, Anti neutrophil cytoplasmic antibody (ANCA), anti-centromere, anti scl-70 and U1RNP were normal. Radiographic skeletal survey showed multilobulated 'cloud-like'soft tissue calcification around joints with normal joint morphology (Fig.1b,c). Sonographically there were no renal and ureteric calculi or nephrocalcinosis. He had undergone excision of a lesion from the axilla before presenting to us, and the histopathology report had shown multiple cysts filled with calcified deposits lined by histiocytes consistent with tumoral calcinosis.

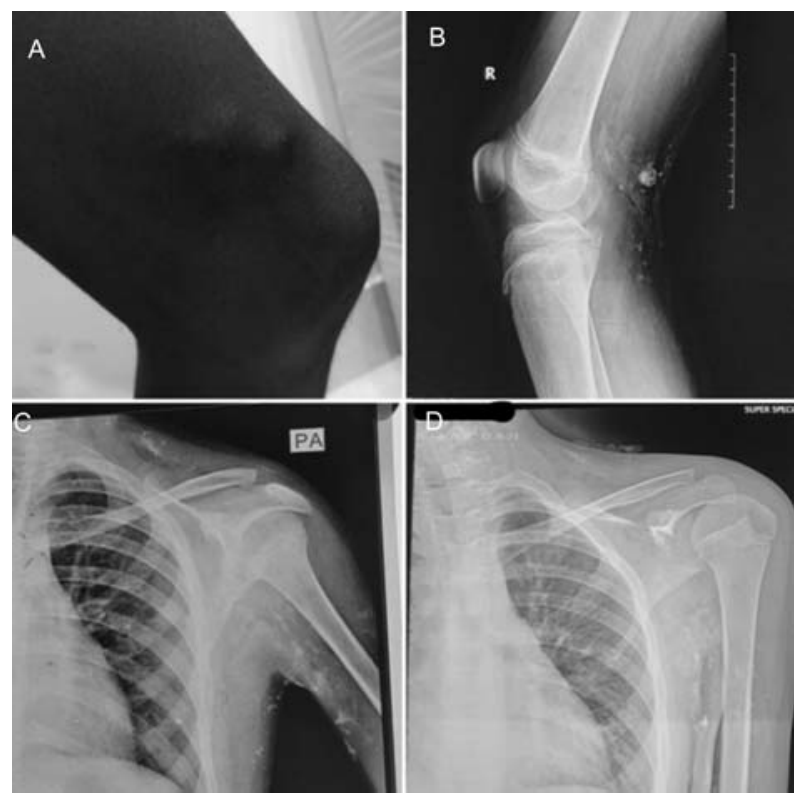

Fig. 1 (a) periarticular hard swellings at right knee, (b) $X$-ray of right knee joint showing multiple "cloud- like" periarticular calcifications, (c) $X$-Ray showing periarticular calcifications at left axilla and neck along platysma, (d) $X$-Ray of left axilla after 6 months of treatment with sevelamer and low phosphorus diet showing decrease in extent of calcification at axilla and neck.

Differential diagnoses considered were disorders which cause dystrophic calcification (like infection, inflammatory processes, cutaneous neoplasm or connective tissue diseases), metastatic calcification (like hypercalcemia or hyperphosphatemia) and secondary tumoral calcinosis due to chronic kidney disease. All these differentials were effectively excluded by evaluation. Final diagnosis of normophosphatemic idiopathic tumoural calcinosis was made.

Multidisciplinary team including endocrinologist, orthopaedician, paediatrician and physiatrist decided to start phosphate lowering medical management aiming to keep phosphorus in the low normal range, with graded physiotherapy and follow up closely after obtaining patients consent. Along with a low phosphorus diet, sevelamer at a dose of $400 \mathrm{mg}$ twice daily was given orally. Surgical correction of deformity was reserved for a later scenario if medical and physical measures failed.

After 6 months of follow upon therapy, he reported improvement in joint movements and limp had disappeared which were confirmed on examination. $X$-rays showed decrease in the size of calcifications at axilla, knee and neck (Fig. 1d), whereas the lesion in the elbows did not increase in size. Smaller lesions have disappeared also. During the one year of follow-up, while maintaining serum phosphorus level between $3.3 \mathrm{mg} / \mathrm{dL}$ and $3.5 \mathrm{mg} / \mathrm{dL}$, he reports no new skin lesions or movement restriction nor any adverse events related to therapy.

Tumoral calcinosis is divided into hyperphosphatemic (familial), normophosphatemic and secondary variants [2]. Hyperphosphatemic form is the most common. High 
phosphorus levels are due to a reduced renal clearance due to decreased action of the phosphaturic hormone fibroblast growth factor 23 (FGF23) which in turn may be due to mutated FGF23 or an enzyme involved in stabilization of wild type FGF23, or $\alpha$-klotho (the cofactor for FGF23 action). Disorders like chronic renal failure with secondary hyperparathyroidism and hyper-vitaminosis D cause the secondary variety. In normophos-phatemic tumoral calcinosis, family history is usually absent, even as recent literature shows emerging evidence of familial basis occurring due to mutations in the gene encoding for the protein sterile alpha motif domain-containing-9 protein (SAMD9) [3]. Normophosphatemic version presents before second decade of life and is associated with tropical or subtropical region of living.

Traditionally, complete surgical excision of symptomatic lesions as and when they appear is the treatment of tumoral calcinosis, but recurrence is the rule. Various methods to lower serum phosphorus have been tried in hyperphosphatemic familial variety, with marked clinical and radiological resolution of lesions and includes the use of aluminium hydroxide, sevelamer, lanthanum carbonate or acetazolamide [4]. Bisphosphonates have also been tried with successful resolution of lesions in some cases [5]. Dietary phosphorus restriction to as low as $400 \mathrm{mg}$ /day is required.

Unlike the hyperphosphatemic variety, the effectiveness of medical therapy in normophosphatemic variety is not established. The only report in literature on medical therapy in normophosphatemic TC is by Jubbin, et al. [6], who described resolution of pain and radiological subcutaneous calcification with alendronate. The present case is the first to report beneficial effect of phosphate lowering therapy in normophosphatemic tumoral calcinosis.

The current case report shows subjective improvement in pain, limitation of movement and gait and an objective improvement in range of movements of joints when phosphate lowering therapy was used with graded physiotherapy in normophosphatemic tumoral calcinosis. Further consideration to phosphate lowering therapy is warranted in children with normophosphatemic tumoral calcinosis.

Sarayu Soumya, ${ }^{1}$ Nandini Prasad, ${ }^{2}$
Puthiyaveettil Khadar JAbBar, ${ }^{1}$ SAJID Hussain, ${ }^{3}$
Chellamma Jayakumari ${ }^{4}$ AND Abilash NaIR ${ }^{{ }^{*}}$
From Departments of ${ }^{1}$ Endocrinologyand
Metabolism, ${ }^{3}$ Orthopaedics, ${ }^{4}$ Internal Medicine,
Government Medical College; Thiruvananthapuram;
${ }^{2}$ Pediatrics, Health Services Department,
Government of Kerala, Kerala, India.
$-{ }^{*}$ abhimck@gmail.com

\section{REFERENCES}

1. Reiter N, El-Shabrawi L, Leinweber B, Berghold A, Aberer E. Calcinosis cutis, part I: Diagnostic pathway. J Am Acad Dermatol. 2011;65:1-14.

2. Smack D, Norton SA, Fitzpatrick JE. Proposal for a pathogenesis-based classification of tumoral calcinosis. Int J Dermatol. 1996;35:265-71.

3. Hershkovitz D, Gross Y, Nahum S, et al. Functional characterization of SAMD9, a protein deficient in normophosphatemic familial tumoral calcinosis. J Invest Dermatol. 2011;131:662-69.

4. Ichikawa S, Baujat G, Seyahi A, et al. Clinical variability of familial tumoral calcinosis caused by novel GALNT3 mutations. Am J Med Genet A. 2010; 152A:896-903.

5. Balachandran K, Kamalanathan S, Sahoo JP, Das AK, Halanaik D. Differential response of idiopathic sporadic tumoral calcinosis to bisphosphonates. Indian $\mathrm{J}$ Endocr Metab. 2014;18:521-25.

6. Jacob JJ, Mathew K, Thomas N. Idiopathic sporadic tumoral calcinosis of the hip: successful oral bisphosphonate therapy. Endocr Pract.2007;13:182-86.

\section{Successful Convalescent Plasma Therapy in a Child With Severe Coronavirus Disease}

Most pediatric coronavirus disease (COVID-19) patients are asymptomatic or have mild to moderate disease and recover within two weeks $[1,2]$. In children, severe acute respiratory distress syndrome (ARDS) can occur, which may progress to toxic shock syndrome. In some affected children clinical features of Kawasaki disease may be observed [3]. Therapeutics like antiviral drugs and/or immune modulators available for COVID19 children have weak recommendations [4]. COVID convalescent plasma (CCP) has been used successfully in the recent global outbreak for the treatment of adult patients with
COVID-19 [5,6]. We report paediatric patient who received CCP as a therapeutic option for treatment of severe COVID-19.

A severely undernourished 13-year-old girl with fever, cough, sore throat for three days was admitted with severe respiratory distress and restlessness. On admission, she was febrile, with tachycardia (146/min), hypotension $(90 / 58 \mathrm{~mm}$ $\mathrm{Hg}$ ) and respiratory rate of $20 / \mathrm{min}$. The oxygen saturation was $88 \%$ on room air. Nasopharyngeal swab reverse transcriptase polymerase chain reaction (RT-PCR) confirmed SARS-CoV-2, and a diagnosis of severe COVID-19 was made. Child was tried with non-invasive ventilation, which was subsequently escalated to pressure control mode of mechanical ventilation. In view of hypotension, cytokine storm was thought of as a possibility and appropriate fluid resuscitation was done. Arterial invasive blood pressure monitoring was done, along with use of inotropic agents like noradrenaline. Bedside echocardiography suggested ejection fraction of $26 \%$. Child was 